\title{
Rollen und Interaktionsmuster ägyptischer Gottheiten in Heilsprüchen als Coping-Strategie
}

\author{
Jana Raffel \\ Universität Leipzig \\ Ägyptologisches Institut
}

\begin{abstract}
Zusammenfassung
Magische und magisch-medizinische Texte stellen einen wichtigen Bestandteil altägpytischer Krankenbehandlung dar. Dieser Beitrag geht auf den Zusammenhang der Religion und Medizin ein und befasst sich mit der Rolle der altägyptischen Gottheiten im Heilsystem Altägyptens. Die Handlungen dieser Gottheiten werden in Bezug auf Coping-Strategien der kranken Menschen und deren religiösen Hintergrund vorgestellt. Zwei Coping-Strategien werden dabei näher beschrieben. Die Abkürzung "p“ in den Quellenangaben verweist auf Papyri.
\end{abstract}

Krankheiten ${ }^{1}$ sind weitverbreitete Universalien, denen es seit jeher entgegenzutreten gilt. Dazu gehört das Heilen von Unwohlbefinden, aber auch die Hilfe zur Bewältigung der Krise (coping). Bei der Bewältigung sind neben dem subjektiven Krankheitserleben und der Reaktion auf die Krankheit gleichfalls die Einflussfaktoren von Bedeutung, die das coping positiv oder negativ beeinflussen können, um adäquate Bewältigungsziele und -formen zu erarbeiten (vgl. u. a. Buddeberg/Willi 1992; Pargament 1997; Pargament, et al. 1988). Im Folgenden wird die altägyptische Medizin vorgestellt und eine Form des religiösen copings sowie die Handlungen der Gottheiten beleuchtet. Die altägyptischen Heilungstexte könnten dabei als eine Form einer altägyptischen Copingstrategie gelten. Diesen Texten widme ich mich in meinem Dissertationsvorhaben.

Das Anliegen meiner Forschung ist die Analyse und Auswertung des Rollenrepertoires und des Interaktionsverhaltens von Götteraktanten innerhalb der Heilungssprüche, um ein Schema der Verwendbarkeit und Verteilung der Götterrollen und -konstellationen in diesem Spruchkorpus aufzuzeigen. Die Rollen können je nach Bedarf angepasst werden, sodass eine Gottheit in ver-

Erschienen in: arbeitstitel - Forum für Leipziger Promovierende, Band 8, Heft 1 (2020), S. 24-33. Lizenzierung CC-BY-SA 4.0.

1 Krankheit im Sinne von Abwesenheit von Gesundheit und Wohlbefinden leiblicher oder seelischer Art. Vgl. Westendorf 2010: 202. Sowie das Vorwort des Bandes von Janowski, B./ Schwemer, D., S. XV. Nach der Satzung der WHO: »ein Zustand vollständigen körperlichen, seelischen und sozialen Wohlbefindens und nicht nur das Freisein von Krankheit oder Gebrechen.«, http://www.euro.who.int/de/about-us/organization/whoworldwide, letzter Zugriff: 05.03.2015. schiedenen oder ähnlichen Situationen unterschiedliche Rollen einnehmen kann. Hier soll der Frage nachgegangen werden, ob und welche Motive bei der Auswahl zugrunde lagen. Die zielsetzenden Fragen dabei lauten:

- Welcher Gott kann welche Rolle bei welchen abzuwendenden Krisen einnehmen?

- Welche Gottheiten treten in ihren jeweiligen Rollen in Interaktion?

- Welche Interaktionen sind das? Wie äußern sie sich?

- Kann eine Verwendungsspezifik der Götterrollen und des abzuwehrenden Unheils nachvollzogen werden?

Die Abgrenzung des Corpus der Heilungssprüche erfolgt aufgrund des Wirkfeldes gegen einen Schicksalsschlag, der die Gesundheit und das Wohlbefinden einer Person in Mitleidenschaft gezogen hat oder ziehen könnte, also reaktiv und präventiv. Grundbedingung der zu untersuchenden Sprüche sind weiterhin die durch den götterweltlichen Präzedenzfall zu identifizierenden Rollen und Interaktionen mehrerer Gottheiten. Als Materialbasis dienen medizinische, sogenannten magisch-medizinische und magische Textquellen ${ }^{2}$ aller Textträgerarten (Papyri, Stelen, Ostraka) vom Mittleren Reich (um 2000 v. Chr.) bis in die saïtisch-persische Epoche (332 v. Chr.) ausgewertet werden. Die Texte der klassisch-pharaonischen Zeit sind als zahlreich und aussagekräftig für die Fragestellung einzustufen, sodass die als zu umfangreich anzusehende Quellenbasis der ptolemäisch-römischen Zeit in dieser Arbeit ausgeklammert bleibt.

Die Themenwahl geht auf meine Magisterarbeit $\mathrm{zu}-$ rück, in der ich mich mit der Rolle des Gottes Horus als Arzt und Patient in den sog. magischen Texten beschäftigt habe (Raffel 2009). Ausgehend von den Beobachtungen meiner Magisterarbeit, in der ich zeigen konnte, dass der Gott Horus in den von mir untersuchten Sprüchen die Rollen des Patienten, des Heilers oder des sich selbst heilenden Patienten übernimmt, werden weitere Rollenzuordnungen und Rolleneigenschaften, sowie deren Interaktion mit anderen Aktanten herausgestellt und verglichen.

Magische Texte wie auch magisch-medizinische Quel-

\footnotetext{
${ }^{2}$ Dieses Quellenmaterial bezieht sich auf die verschriftlichte Form der Hoch- bzw. Königsreligion (vgl. Fitzenreiter 1994: 52). Welche Anteile an »Volksglaube« bzw. "persönlicher Frömmigkeit« hineinspielt, muss je nach Textaussage von Fall zu Fall geprüft werden. Wenn verallgemeinerte Aussagen gemacht werden, werden sie auf dieser Grundlage getroffen.
} 
len wurden unter unterschiedlichen Fragestellungen bearbeitet, jedoch fehlt bisher eine eingehende Analyse der götterweltlichen Aktionen und Handlungen. Die Aufarbeitung dieses Desiderats bildet das Ziel des Dissertationsvorhabens und wird hier in Ansätzen näher vorgestellt.

\section{Quellenlage}

Das heutige Wissen über die altägyptische Medizin speist sich aus einem Corpus medizinischer bzw. magisch-medizinischer Papyri mit Traktaten zu Gynäkologie, Hautkrankheiten, Gefäß- und Muskelbehandlung, einer Sammelhandschrift zur Traumatologie sowie einer Rezeptsammlung mit 880 Diagnosen und Rezepten auf knapp 19 Meter Papyrus, dem Papyrus Ebers (vgl. Fischer-Elfert 2007). Einige dieser Schriften, wie der Papyrus Smith beinhalten unter anderem Anweisungen, wie ein Patient untersucht werden soll und welches Leiden mit Hilfe der Untersuchung festgestellt wird, gefolgt von der Therapie. Das klassische Schema ist etwa: »Wenn du (= behandelnder Heiler) einen Mann/Patienten untersuchst/ beurteilst/betrachtest und er an (Befund) XY leidet/XY hat und du vorfindest (die Symptome) XY, dann sollst du dazu sagen: Der Befund XY ist es, eine Krankheit, die ich behandeln werde/mit der ich kämpfen werde/die man nicht behandeln kann.« Abgeschlossen wird diese Diagnose mit der Therapie bzw. dem Rezept: »Dann sollst du verbinden/herstellen etc.«. In einigen Fällen kann am Ende der Rezeptur angemerkt sein: »Wirklich vorzüglich; unzählige Male/millionenfach erprobt«.

$\mathrm{Zu}$ den nichtmedizinischen Quellen im eigentlichen Sinne werden Orakeldekrete, so genannte magische Texte mit Beschwörungen, literarische Quellen, Briefe an Tote, Nachrichten über Ärzte oder ausländische Schriftzeugnisse (Herodot, Diodor, akkadische Quellen usw.) gezählt (vgl. Westendorf 1999: 5f.). Weitere Quellen sind Stelen und Grabinschriften oder Plastiken, durch die ca. 150 Ärzte vom Alten Reich bis in das 1. Jahrhundert vor Christus bestimmt werden konnten (vgl. Ghalioungui 1983, Halioua/Ziskind 2005: 14; Fischer-Elfert 2007: 51). Weitere archäologische Funde sind Mumien, Grabfunde wie Instrumentarien von Ärzten (Messer, Skalpelle usw. (vgl. Westendorf 1999: 486ff; Halioua/Ziskind 2005: 34.) oder Sanatorien. Weiterhin kommt der Aspekt der Paläopathologie sowie der Mumienforschung hinzu. ${ }^{3}$

\section{Heilsystem I}

Medizin (von lat. mederi: heilen) ist ein Heilverfahren, das zur Genesung beitragen soll. Nach Karl Hoheisel sind unter dem Begriff »nichtmedizinische« Heilsysteme »alle Verfahren, die im naturwissenschaftlichen Paradigma nicht zu erklären sind « (Hoheisel 1995: 171) zu verstehen. Nach dieser Definition treten also in der »Altägypti-

\footnotetext{
${ }^{3}$ Nach Fischer-Elfert 2007: 43 »stagniert immer noch die Kommunikation zwischen der naturwissenschaftlichen und der philologischen Interpretation, auch kommt es bisweilen zu vorschnellen paläoanthropologischen Bestimmungen anhand von unkritisch übernommenen, sprachwissenschaftlich begründeten oder im Unklaren belassenen Identifikationen dieser oder jener Krankheit.«
}

schen Medizin« medizinische und nichtmedizinische Heilverfahren nebeneinander.

In der ägyptologischen Forschung wird beim altägyptischen Heilwesen zwischen empirisch-rationaler versus magisch-religiöser Medizin unterschieden (vgl. Kolta/ Schwarzmann-Schaffhauser 2000; Leitz 2002b.). Diese Trennung ist im Bereich der ägyptischen Medizin allerdings schwierig, da sie nicht der altägyptischen Praxis entspricht. Als Beispiel sei hier ein Zitat aus dem medizinischen Papyrus Ebers angeführt, das genau diese Verbundenheit beider Anwendungsweisen verdeutlicht:

»Stark (nht) ist die Magie (hk3.w) wegen der Medizin (phr.t), (und) stark ist die Medizin wegen der Magie. [...] Ein wahres Heilmittel, millionenfach erprobt.« (pEbers Spruch 3, rt. 2,22,3, zitiert nach Fischer-Elfert 2005a: 143-144.)

Die durch den Erhaltungszufall medizinischer Papyri bedingte Annahme, dass sich der Bereich der rationalen Medizin vorwiegend im Alten und Mittleren Reich abgespielt habe, die Magie sich jedoch erst ab dem Neuen Reich etablierte, wird heute nicht mehr vertreten. ${ }^{4}$

\section{Exkurs zur Magie}

Das Konstrukt oder Konzept der Magie wurde durch christliche Prägung oft als negativ oder gar als (minderwertiger) Gegensatz zur Religion verstanden und als Aberglaube abgetan (Erman 1934: 296f.). Magie im altägyptischen Kontext muss als ein fester Bestandteil des ägyptischen Weltbildes (Schneider 2000: 47; Stegbauer 2015: 37), als »ein umfassendes System zur Stabilisierung der bestehenden Ordnung« (Schneider 2000: 37) verstanden werden und ist laut der viel zitierten Lehre für Merikare den Menschen gegeben worden, sich zu verteidigen (vgl. Quack 1992: 79). Der ägyptische Begriff ḥk3 (Heka), der mit Magie übersetzt wird, ist nicht mit unserem heutigen, meist christlich geprägten Verständnis von Magie gleichzusetzen (Stegbauer 2015: 37 und 10-12; Gutekunst 1987: 94 und Ritner 1993: 236). Die altägyptische Magie wird als Naturgesetz (Vgl. Stegbauer 2015: 37-38; Ritner 1993: 8 u. 249; auch Schneider 2000: 44 46 und 81) verstanden. Heka kann nach der Auffassung von Schneider in einer ersten Annäherung als eine Schöpferkraft definiert werden, »ohne die die Welt nicht entstanden wäre und nicht weiter bestehen könnte«. (Schneider 2000: 45) Sie wirkt also als »kreative Potenz«, die zeitweise personifiziert sowie kultisch verehrt wurde (Vgl. Fischer-Elfert 2006, 1.1). Als Naturgesetz muss Heka als rational angesehen werden, ist weder Glaube noch Aberglaube und nicht von bestimmten Lebensbereichen altägyptischer Menschen abzugrenzen. Das heißt, dass die Magie alle Sphären der altägyptischen Lebenswelt im Diesseits und Jenseits umfasste; jedoch nicht, wie dies gerne getan wurde, von der Religion oder

\footnotetext{
${ }^{4}$ Die Annahme gründet auf dem hohen Anteil an sog. Zaubersprüchen in den Papyri des Neuen Reiches und dem vermeintlich empirischen Papyrus Smith. Vgl. z. B. Westendorf 1992: 19-21. Zu gegenteiligen Aussagen vgl. Kolta/SchwarzmannSchaffhauser 2000: 65 und Leitz 2002b: 50.
} 
Medizin (Vgl. Westendorf 1992: 19-26 und Westendorf 1999: 3) zu trennen ist. Diese Grenzziehung, die dennoch weiterhin in der wissenschaftlichen Forschung betrieben wird, erfolgt »demnach mehr aus praktischen Gründen denn aus kulturimmanenten « (Stegbauer 2015: 37; vgl. auch Schneider 2000: 38-39; Fischer-Elfert 2006, 2). Heka ist moralisch neutral und wird erst in der Anwendung für oder gegen eine Person als positiv oder negativ bewertet (Vgl. Ritner 1993: 20).

\section{Heilsystem II}

Eine Gesellschaft bringt Vorstellungen und Institutionen hervor, die ihrer Umwelt entsprechen. Berger erklärt in seinem Artikel das Verhältnis von Religion und Welterrichtung und geht dabei auf die Dialektik der Gesellschaft ein (vgl. Berger 1973: 3-28). So sind desgleichen die Methoden der altägyptischen Medizin ein Produkt der Menschen, ihrer Vorstellungen vom Menschenbild und dem Kosmos, in dem sie lebten. Zu diesen Vorstellungen gehören die religiösen Komponenten, sowie der Wissensstand über den Körper und die Auffassung von Krankheitsursachen und deren mögliche Behandlung.

Die überlieferten Krankheiten werden heute in endogene versus exogene Krankheitsbilder eingeteilt (vgl. dazu Kolta/Schwarzmann-Schaffhauser 2000: 57). Unter endogen werden durch innere Ursachen bedingte Erkrankungen verstanden. Im Alten Ägypten waren die Verursacher meist Dämonen oder Wiedergänger ${ }^{5}$, die durch Körperöffnungen (meist linke) von außen eindringen. ${ }^{6}$ Eine weitere Krankheitsursache wird der Nahrung zugeschrieben. So sollten durch unverdaute oder schlechte Nahrung Schleim-, Eiter- oder Schmerzstoffe gebildet werden und zu Schwellungen, Geschwüren oder Ausschlägen führen. Als exogene Krankheiten gelten äußere Verletzungen wie Brüche, offene Wunden, Bisse und Stiche.

Die anatomischen Kenntnisse werden von Kolta und Schwarzmann-Schaffhauser als "verschwindend gering " eingeschätzt, wobei jedoch die »differenzierten, empirisch-rationalen Vorgehensweise[n] bei der anatomischen Lokalisation, Diagnose und Therapie verletzungsbedingter äußerer Erkrankungen« dazu im Widerspruch stehen (Kolta/Schwarzmann-Schaffhauser 2000: 87) ${ }^{7}$. Das Messen des Pulses scheint den altägyptischen Ärzten vertraut gewesen zu sein (vgl. pSmith Fall 1, Glosse A). Allein diese Aussagen zeigen, dass eine Einzelfallentscheidung und genaue Überprüfung der Quellen notwendig ist, um die genauen Fachkenntnisse altägyptischer Heiler einschätzen zu können. Eine Schlüsselrolle wurde dem Her-

\footnotetext{
${ }^{5}$ Wiedergänger sind verklärte Tote (Un-Tote), die durch nicht ordnungsgemäße Bestattung die Lebenden heimsuchen. Auch Ach-Geister, wie in der Lehre des Ani B 21,20-22,3 (nach Fischer-Elfert 2005b, Nr. 19) können aus Rache für zu wenig Kulterhalt, ihre Nachfahren belästigen (a. a. O, S. 28).

${ }^{6}$ Kolta/Schwarzmann-Schaffhauser 2000: 57 geben ebenso das Beispiel der Verstopfung als endogene Ursache an, die nicht dämonischen Ursprungs ist, sondern ernährungsbedingt verstanden wird. Vgl. weiter Leitz 2002b: 50; Leitz 2005: 43.

${ }^{7}$ Zur grundlegenden Kritik an der Vorgehensweise und fehlender Beachtung wichtiger ägyptologischer Literatur siehe Quack (2003) und Pommerening (2005).
}

zen beigemessen. Dieses galt als der Sitz des Verstandes, womit das Gehirn als für diese Funktion unwichtig angesehen wurde und dem bei der Balsamierung keine weitere Bedeutung zufiel. Das Gehirn wird als Abfallprodukt aus dem Körper entfernt, während das Herz darin verbleibt. Es wird zum Teil separat einbalsamiert und zurück in den Körper gelegt, um dem Toten mit auf den Weg ins Jenseits gegeben zu werden. Hier kommt die Vorstellung des Wägens des Herzens gegen die Feder der Maat beim Totengericht zum Tragen. Gefäße (mtw), die nach Kolta und Schwarzmann-Schaffhauser allerdings nicht mit einem »primitiven Kreislaufmodell« interpretiert werden dürfen, transportieren Wasser und Luft zu jedem Körperteil, wobei keine klare Unterscheidung zwischen Herz und Verdauungstrakt vorhanden gewesen sein dürfte (vgl. Kolta/Schwarzmann-Schaffhauser 2000: 90-92). Es wurde als ein körpereigener Versorgungs- und Ableitungsstrom gedacht, dessen geregelte Funktion als entscheidend für das Wohlbefinden galt (Vgl. Kolta/Schwarzmann-Schaffhauser 2000: 89). ${ }^{8}$ So galten Verstopfungen als Krankheitsursache, die durch »Verlegung eines Stromlaufes durch ein Hindernis mit konsekutivem Rückstau vor und Mangelversorgung nach dem Hindernis [...], zu Beschwerden in weit entfernten Körperteilen führen, etwa zu Schwellungen im Rücken, zu entzündeten Augen oder zu einer laufenden Nase« (Kolta/SchwarzmannSchaffhauser 2000: 94-95). Umherziehende Schmerzstoffe (whdw) und Schleimstoffe (st.t) können ebenfalls eine Folgeerscheinung der Verstopfung sein und sich durch den gesamten Körper verbreiten. Das Blut wurde laut Kolta und Schwarzmann-Schaffhauser nur als Ausscheidung des Körpers angesehen (vgl. Kolta/Schwarzmann-Schaffhauser 2000: 94).

Weitere Krankheitsverursacher werden in vielen der Sprüche direkt angesprochen als Feinde, Götter/Göttinnen, Dämonen/Dämoninnen und Wiedergänger/Wiedergängerinnen oder sogar als Ausländerin (Asiatin oder Nubierin) $)^{9}$. Diese dringen häufig des Nachts oder an besonders »gefährlichen« Tagen auf Eigeninitiative oder auf Geheiß von Göttern durch Körperöffnungen in den Patienten ein. ${ }^{10}$ Auch die Aussage: $» k$ krank vor Liebeskummer « wird in den literarischen Quellen betont. ${ }^{11}$

Die Heilungsmethoden werden, wie oben schon angeführt, gern in empirisch-rationale und magisch-religiöse unterteilt. Krankheiten werden beseitigt, entfernt, herausgeholt, abgewehrt, vernichtet oder getötet. ${ }^{12}$ Dem Heiler stehen dazu zum einen die mechanischen Vorgehensweisen zur Verfügung, d. h. Verbinden, Operieren, Massieren, Verabreichen von Klistieren, u. a. Eine weitere Me-

\footnotetext{
${ }^{8}$ Diese Vorstellung wird als »naturphilosophisch« verstanden, deren Ebenbild der Nil sein soll. Vgl. Westendorf: 1992: 44.

${ }^{9}$ Zaubersprüche für Mutter und Kind. Schneider 2000: 48; pLondon; Fischer-Elfert 2007: 46, wohl aus Asien stammende Krankheit (evt. Lepra lepromatosa).

${ }^{10}$ An den Epagomenentagen wurden besonders die Boten der Sachmet gefürchtet, die durch ihren Hauch oder ihre Pfeile die so genannte Seuche des Jahres verursachen.

11 Vgl. die sogenannten Liebeslieder z.B. des pHarris $500=$ pBM EA 10060 oder pChester Beatty I, Verso.

${ }^{12}$ Zum Ägyptischen Vokabular s. Westendorf 1999: 483-484.
} 
thode ist die auf der Ernährung beruhende. Dazu zähle ich die Verordnung von Diäten ${ }^{13}$ und Einnahme von Drogen (Tees, Pillen etc.). Nach den in den Papyri aufgelisteten Rezepten mit genauen Quantitätsangaben konnten annähernd hundert Vokabeln zu den Tätigkeiten der pharmazeutischen Drogenbereitung und Verabreichung festgestellt werden. So konnten Pflanzen, Mineralien oder tierische Produkte ${ }^{14}$ zerrieben, zerquetscht, zerteilt, aufgekocht, verbrannt, verdünnt, gekaut, geschluckt, gegessen, verräuchert oder als Klistier genutzt werden. ${ }^{15}$ Die Konzepte zur Auswahl der religiös wirkenden Objekte in der altägyptischen Medizin folgen dem Prinzip der Identität, der Farb-, Form- oder Handlungsähnlichkeit oder der Aneignung von Simile-Kräften. ${ }^{16}$

Als magisch-religiös bezeichnete Methoden zählen Sprüche, die Analogien zu den Mythen, vor allem um Isis, Osiris, Horus und Seth ziehen. Die altägyptische Götterwelt war allgegenwärtig und aktiv am Schicksal der Menschen beteiligt. ${ }^{17}$ Sie durchzog die gesamte Lebens- und Denkwelt - so auch den medizinischen Bereich. Demnach wurden Götter um Hilfe und Beistand gebeten oder bedroht, wenn sie für die Krankheit verantwortlich waren und diese nicht rückgängig machen wollten. Die Androhungen waren dann derart, dass sie u. a. keine Riten und Opfer mehr erhalten sollten, ihre Schreine geschändet würden, die Welt zum Stillstand gebracht werde (zuletzt Raffel 2019). Bekannt ist weiterhin das so genannte »Sündenbockprinzip « ${ }^{18}$, bei dem z. B. die Tollwut eines Menschen durch das Entlangstreichen von Pflanzen an der verursachenden Bisswunde von diesen aufgenommen werde, um sie dann wieder auf einen Hund zu übertragen (vgl. Leitz 2002b: 53). Innerhalb eines religiösen Textes wird kein Element zufällig gewählt, sondern jede Person, Handlung, jedes Objekt oder Wort wird sorgfältig komponiert und steht in streng logischer Beziehung (vgl. Leitz 2002b: 52).

Da Magie in Wort, Bild (vgl. Eschweiler 1994) und Tat wirkt, ist eine ausführende Handlung der den Sprüchen innewohnenden Geschichte oder Anweisung immer mitzudenken. Magisch-religiöse Objekte, die Verwendung fanden, sind etwa Heilstatuen und Horusstelen (vgl. Sternberg-el Hotabi 1999, Kessler 2005). Diese vor

\footnotetext{
${ }^{13}$ Leitz 2002b: 52 sieht in diätischen Vorschriften, z. B. einen Fisch zu essen, eher einen kultischen Hintergrund und »eigentlich nie einen medizinischen «, da diese öfter in religiösen Texten auftreten. Diese Behauptung sollte meines Erachtens jedoch nicht für allgemeingültig genommen, sondern je nach Fall geprüft werden.

${ }^{14}$ Dreck- und Tabunamen sind noch nicht geklärt, vgl. FischerElfert 2007: 50. Quack 1996 konnte unter dem Namen »Pavianshaar« den Dill identifizieren. Vgl. auch Vorschlag von Leitz 2005, Analogien für die Verwendung der Zutaten aufzuzeigen.

${ }^{15}$ Weitere Beispiele siehe Leitz 2002a: 28-29.

${ }^{16}$ Für Beispiele der Anwendung vgl. Leitz 2005: 44-45.

${ }^{17}$ So wurde häufig in Briefen geäußert: »Das Morgen liegt in der Hand Gottes [...]«; dazu siehe: Wente 1967. Zur Gottesnähe siehe Assmann 1991, Kap. 1.

${ }^{18}$ Zur theoretischen Grundlage des Sündenbockprinzips siehe Girard 1998 und zur Anwendung der Theorie in der Ägyptologie vgl. Rost 2008.
}

allem in der ägyptischen Spätzeit verwendeten Gegenstände lehnen sich an den Mythos um Horus an. Die Heilwirkung dieser Stelen erfolgt durch ihre Berührung oder durch das Übergießen mit Wasser und das darauffolgende Anwenden innerhalb eines Tranks, einer Salbe o. ä.

Weitere sehr beliebte apotropäische Objekte waren Amulette. Eine Kategorisierung von Amuletten nach ihrer Funktion wird unterschieden in »homopoeic (similars) «: der Träger wünscht sich die Eigenschaften, die der dargestellte Gegenstand verkörpert; »phylactic (protection)«: Amulette, die mit Mythen oder Göttersymbolen in Verbindung stehen, verleihen Schutz; »theophoric (gods)«: Bilder von Göttern erbitten deren Beistand; »dynatic (powers)《: Machtsymbole verleihen Kraft; »ktematic (property)«: Gegenstände sichern Besitztümer. ${ }^{19}$ Jedoch haben nur die ersten drei in der medizinischen Anwendung Relevanz. Als Amulette werden weiterhin Papyrusstreifen verstanden, die um den Hals getragen worden sind und auf denen dann ein Spruch und das anzuwendende Symbol geschrieben stehen. ${ }^{20}$

Die Auswahl der heilsam wirkenden Objekte (sowohl in der Drogenzusammenstellung als auch bei Amuletten) in der altägyptischen Medizin folgen zum Teil den Prinzipien der Identität, der Farb-, Form- oder Handlungsähnlichkeit, oder der Aneignung von Simile-Kräften. ${ }^{21}$

\section{Coping-Strategie I}

Krankheiten und z. T. deren Ursachen können als so genannte Stressoren eine negative Auswirkung auf den Genesungs- und Heilungsprozess haben oder sogar mit ungesunder psychopathologischer und psychogener Störung einhergehen. »Die so entstehenden vielfältigen psychosozialen Belastungen sind jenen, die durch andere Stresszustände bedingt sind, durchaus vergleichbar. Immer muß der Patient in irgendeiner Form auf seine wie immer beschaffene Krankheit reagieren.« (Buddeberg/Willi 1992: 483) Diese Belastungen äußern sich in körperlichem Unwohlsein, indem sie subjektive Angstaffekte, Gefühle von Verkrampfung, Beklemmung oder Schmerz, Schlaflosigkeit, Schwindelgefühl, Selbstbewusstseinsstörungen hervorrufen. ${ }^{22}$ Ebenso können mögliche soziale Ausgrenzung oder existentielle Belastung ${ }^{23}$ zu einem gestörten emotionalen Gleichgewicht führen. Ausgrenzung durch eigene Schuld am Leiden (Strafe Gottes) aufgrund von negativen moralischen Verhaltens kann den Leidensdruck erhöhen. Die Heilung wird auf göttliche Gunst zurückgeführt, die den Patienten wiederum in seine soziale Stellung in der Gesellschaft stärkt (Vgl. Hoheisel 1995: 183). Bestimmte Krankheiten wie Aussatz schienen zur Folge $\mathrm{zu}$ haben, dass derjenige von der Gesellschaft und damit

\footnotetext{
${ }^{19}$ Kategorisierung nach Petrie s. Schneider 2000: 72, Fn. 109.

${ }^{20}$ Eine grundlegende Bearbeitung und Zusammenstellung der sog. Oracular Amuletic Decrees (OAD) gibt Edwards 1960. Weitere Beispiele bei Burkard 2006 oder Fischer-Elfert 2015.

${ }^{21}$ Für Beispiele der Anwendung vgl. Leitz 2005: 44-45.

${ }^{22}$ Vgl. Haas 2002: 25f.

23 Die existentielle Belastung meint die möglichen Versorgungsengpässe der Familie durch Abwesenheit bei der Arbeit oder dadurch bedingte schlechte Ernten.
} 
auch von der Familie getrennt und in eigene Gebiete am Rand der Wüste geschickt wurde. Kranke wurden als kultisch unrein empfunden und vom Kultgeschehen fern gehalten (vgl. Fischer-Elfert 2005c. Zu Ausgrenzung von Kranken im Tempeldienst: Quack 2005: 64). Für den Verlauf des Genesungsprozesses ist daher eine aktive Krankheitsbewältigung von Vorteil. Das Ziel einer Krankheitsbewältigung ist es, das Individuum auf eine neue, durch die Krankheit ausgelöste Situation einzustellen und ihm zu helfen, mit dieser optimal umzugehen, um eigene Selbstheilungskräfte zu aktivieren, die Mithilfe des Patienten im Genesungsprozess zu sichern und Vertrauen sowie eine positive Grundeinstimmung auf eine Heilung zu fördern. Dies geschieht, indem sich der Patient den erfahrenen Händen eines altägyptischen Heilers überantwortet, der dann mittels Wort oder Tat der Krankheit und den Krankheitsverursachern entgegentritt.

Moderne Studien ${ }^{24}$ zeigen, dass die Religiosität oder Spiritualität eines Menschen in einem engen Zusammenhang mit dessen mentaler Gesundheit oder Bewältigung von negativen Ereignissen steht. Dabei kann die Korrelation sowohl positive als auch negative Auswirkungen haben. Positive Effekte sind: bessere mentale Gesundheit, größeres Wohlergehen, höhere Lebensqualität sowie geringere Raten an Depressionen und Ängsten zu leiden (vgl. Weber/Pargament 2014: 358). Patienten mit religiösem Hintergrund können meist besser mit schwierigen Diagnosen umgehen oder diese bewältigen. Zur spirituellen Unterstützung zählen die positive Bewertung von Stressoren und spirituelle Verbundenheit nicht zuletzt mit der religiösen Gemeinschaft. Der Glaube sowie die religiösen Erklärungen/Antworten, die sich daraus ergeben, können den Patienten helfen, ein gewisses Gefühl von Selbstkontrolle und Hoffnung zu etablieren (vgl. Weber/ Pargament 2014; Harrison u. a. 2001: 87). Spiritueller Druck hingegen kann das Auftreten von Depressionen, Ängsten, Schuldgefühlen und suizidalen Tendenzen unterstützen, negatives Coping korreliert mit geringerer mentaler Gesundheit und Druck (vgl. Pargament et al. 2006: 1161). Des Weiteren können Glaubensgrundsätze die Entscheidungen für oder gegen medizinische Behandlungen beeinflussen bzw. zu Konflikten führen und letztendlich jene Erfolgschancen erhöhen oder minimieren. Ebenso, wenn durch die Glaubensgrundsätze eine Bestrafung Gottes für eine Erkrankung verantwortlich gemacht wird und dies zu einem Rückzug der Gemeinschaft von dem Erkrankten führt, hat dies negative Auswirkungen auf den mentalen Gesundheitszustand des Patienten, was langfristig zu einer körperlichen Verschlechterung führen kann (vgl. Weber/Pargament 2014: 358). Ein gemeinsamer religiöser Hintergrund führt hierbei zu einer verstärkten Vertrauensgrundlage zwischen Patienten und Heiler.

\footnotetext{
${ }^{24}$ In den letzten Jahrzehnten hat die Anzahl der Studien in dem Bereich enorm zugenommen. Hierunter laufen Studien, die sowohl positive wie negative Effekte auf die generelle Gesundheit untersuchen, aber auch Spezialuntersuchungen, die die Wirksamkeit religiösen Copings bei Patienten mit z. B. Krebsdiagnosen oder nach Herz-Operationen beleuchten. Weiterhin werden Studien mit unterschiedlichen Religionsgruppen vorgenommen. Zusammenfassende Überblicke geben Harrison et al. 2001 und Weber/Pargament 2014.
}

Aus diesem Grund ist eine Einbindung religiöser Ressourcen des Patienten wichtig für den Erfolg der Behandlung (vgl. Weber/Pargament 2014: 358).

Somit haben nicht nur die Fähigkeiten und Fertigkeiten des Heilers bei mechanischen Praktiken psychologisch beruhigende Wirkungen, sondern ebenso die als magischreligiös bezeichneten Methoden. Im alten Ägypten kamen Sprüche zum Einsatz, die z. T. als Spruchsammlungen (Heilungstexte) vorliegen. ${ }^{25}$ Sie dienen dazu, den Patienten mittels götterweltlicher Hilfe reaktiv zu heilen oder präventiv vor Krankheiten und Unheil zu bewahren. Im Fall des reaktiven Schutzes kommt eine Historiola (vgl. Frankfurter 1995; Stegbauer 2015: 75-78) zum Tragen, die den Patienten mit einem götterweltlichen Präzedenzfall in Verbindung bringt, um ihm zu helfen. Dabei sind die Aktanten Gottheiten bzw. menschliche Pendants, die als Gottheiten agieren. Der Präzedenzfall par exellence ist dem Osirismythos entlehnt, zu dessen zentralen Figuren Isis, Horus, Seth und Osiris selbst gehören. Der kindliche Horus und Erbe seines Vaters Osiris wird von seiner Mutter Isis vor den Nachstellungen seines Onkels und Vatermörders Seth versteckt. Dort ist er jedoch weiterhin Gefahren wie z. B. Schlangenbissen, Skorpionstichen oder Bauch- und Kopfschmerzen ausgesetzt, die dann wiederum durch göttliche Hilfe geheilt werden. Mittels Analogie (Vergleichen) zu den Mythen, wird dieser zeitenthobene, götterweltliche Krankheits- und Genesungsfall auf den menschlichen Patienten übertragen, der dann ebenfalls Heilung erfährt bzw. erfahren soll.

\section{Exkurs Heilungstexte}

Das Problem der sog. magischen Texte ist ihre Einteilung bzw. Kategorisierung. Diese »beinhaltet verschiedene Abgrenzungsschwierigkeiten« (Gutekunst 1995: 10), die anhand einer systematischen Analyse der sprachlichen Struktur der Texte erfolgen müsste und bisher nur in Ansätzen vorliegt (Gutekunst 1995: 11; Stegbauer 2015: 3940). Die Heilungstexte, die der Spruchliteratur zugeordnet und dadurch Zaubersprüche genannt werden, wurden im 19.-20. Jahrhundert von der ägyptologischen Forschung als »Restkategorie« subsumiert, was bislang verhinderte, »das Corpus der magischen Texte als solches zu fassen: $\mathrm{Zu}$ viele unterschiedliche Textsorten wurden dem Corpus zugerechnet. Gemeinsamer Nenner war immer die vermeintliche Verwendungssituation im privaten Raum, den man von einem vermeintlich offiziellen Rahmen (Tempelkult) und von der Verwendung für Tote abtrennte, eine Unterscheidung, die [...] auf Gardiner zurückgeht.« (Stegbauer 2015: 40, Anm. 212). ${ }^{26}$

Eine Einteilung der Texte in Machtzauber, Schadenszauber und Schutzzauber durch Altenmüller (1980, Sp. 1153-1154) oder nach Borghouts (1980, Sp.11421146) »productive«, »destructive «, »divination « und »de-

\footnotetext{
${ }^{25}$ Zur Geheimhaltung der Texte und zu Geheimformeln siehe: Morenz 1997; Schneider 2000: 57 sowie Ritner 1993: 203-207. Weiterhin zu Laienärzten Kolta/Schwarzmann-Schaffhauser 2000: 159-158.

${ }^{26}$ Vgl. auch Altenmüller 1980, Sp. 1151-1152, der die Schwierigkeit erwähnt, die Texte, die im engeren Sinne als magisch bezeichnet werden können, aus der »Masse« herauszulösen.
} 
fensive magic«, wird von Stegbauer (2015: 47-48) als subjektive Unterscheidung mit Verwendung schwammiger Begriffe ohne Fundierung der Systematik heftig kritisiert. In der neueren Literatur ${ }^{27}$ wird Magie hingegen eingeteilt in: a) präventiv/prophylaktisch, b) kurativ/reaktiv, c) divinatorisch/prognostische/investigativ, d) transformativ. Mit dieser Einordnung wird die nicht sichtbare Aktantenintention umgangen und die zeitliche Wirkebene zum Schicksalsschlag bzw. das Ergebnis herangezogen (vgl. Stegbauer 2015: 48). Überschneidungen der einzelnen Sprüche sind hierbei unumgänglich. Die überkulturelle Textsorte der magischen Sprüche wird in der Ägyptologie von Stegbauer (2015: 47) folgendermaßen eingeordnet: Die Sprüche stehen neben Hymnen, Gebeten, Verklärungen usw., obwohl sie diese beinhalten können $^{28}$, als Untergruppen der Spruchliteratur. Diese können sich dann wiederum, wie oben erwähnt, in präventiv, reaktiv, transformativ und investigativ unterteilen.

Die Sprüche sind nach Spruchtitel/Überschrift, Textteil und Nachschrift/Ausführungsanweisung aufgebaut und in der Praxis immer im Ritualkontext zu sehen (vgl. Ritner 1993: 35-57, Fischer-Elfert 2006, unter 1.2.1.). Das heißt, dass wohl nur der eigentliche und im Gegenteil zur Überschrift und Nachschrift obligatorische Textteil per Wort oder Tat ausgeführt/vorgeführt wurde. Dieser kann verschiedene andere Textsorten aufnehmen, so z. B. Hymnen, Litaneien oder Gebete, die in Gestalt von Anrufungen, Befragungen, schmeichelnden Bitten, Überredungen, Befehlen, auch Beleidigungen oder Bedrohungen gehalten sein können. So trägt Stegbauer folgende »Merkmale der Textsorte `Zauberspruch«« (Stegbauer 2015: 54-73) zusammen, die sie nach Sprechhaltung, interpersonelle Form und Konversationsstruktur gliedert.

Die Sprechhaltung ist in narrative Textabschnitte sowie appellative/informative Textabschnitte unterteilt, die innerhalb der einzelnen Sprüche wechseln können. Der narrative Teil wird von der so genannten Historiola gebildet (vgl. Frankfurter 1995 und Stegbauer 2015: 75-78). Die Historiola ist eine Erzählung, deren Erzählzeit in der Vergangenheit liegt und eine spezifische Ausformung eines Mythos ${ }^{29}$ darstellen kann. Sie kann auf eine bekannte Geschichte zurückgehen, muss dies aber nicht zwangsläufig. ${ }^{30}$ Die durch göttliche Aktanten geprägte Historiola

\footnotetext{
${ }^{27} \mathrm{Zu}$ den nachstehenden Begriffen siehe Schneider, Analogie, 2000 nur mit der Einteilung präventiv und reaktiv; Fischer-Elfert 2006; Stegbauer 2015: 48-49.

${ }^{28}$ Dies ist als Zeichen der Intertextualität anzusehen. Vgl. Stegbauer 2015: 47.

${ }^{29}$ Nach Frankfurter (1995: 474) wird der Mythos als »pre-articulate level, a condensation of social structure, morality, and existential concerns at the broadest level« gesehen. Assmann 1977: 43 spricht der Götterwelt der Pyramidentexte »den Charakter des Mythischen im engeren Sinne noch ab« und möchte die Mythenbildung erst im Mittleren Reich ansetzten. Zur Definition des Mythos als funktionalistischen, religionswissenschaftlichen Begriff als »fundierende, legitimierende und weltmodellierende Erzählung« (S. 180) und »Mentalitätsform bzw. Bewusstseinsstufe« (S. 190), vgl. Assmann 1998: 179-200.

${ }^{30}$ Dazu Frankfurter 1995: 472: »[...] the historiolae >are the myths, rather than derivates of them. « Ebenfalls Stegbauer sieht den angestammten Ort der Mythen in den Historiolae der Sprü-
}

kann dialogische Form annehmen (Stegbauer 2012: 172 184). So wird eine Handlung z. T. mit Monologen und Dialogen entwickelt, die einen "vermittelnden fiktiven textimpliziten Erzähler« (kursiv im Original, Stegbauer 2015: 55) aufweist. Bei der appellativen/informativen Sprachhaltung wird ein vom Sprecher angesprochener Adressat über einen Zustand informiert oder es wird an ihn appelliert, bestimmten Aufforderungen nachzukommen. Dabei können Charakterisierungen (Selbst-, Fremd-, oder Adressatencharakterisierungen), Anrufungen/Invokationen, Schimpfkanonaden, Götterbedrohungen (vgl. Altenmüller 1977; Manisali 2004: 301-309), (Ver)wünschungen oder Gliedervergottungen auftreten (Näheres vgl. Stegbauer 2015: 58-65). Die interpersonelle Form eines Textes macht dabei die möglichen Beziehungen zwischen Sprecher, Adressat und Thema deutlich (vgl. Assmann 2003: 324-325; Assmann 2002: 29-33; mit Ergänzungen Stegbauer 2015: 66-67).

Beschwörungen wirken mittels Analogie. Schneider beschreibt diese als eine Fessel, die »aktuelle Fälle einem verborgenen Regelwerk« (Schneider 2000: 61) unterwirft. Die Welt besteht im altägyptischen Denken aus Chaos „Isfet“" (isf.t) und Ordnung „Maat“ (m3“.t). Die geordnete Welt muss immer wieder in diesen Zustand versetzt werden, da sich das Chaos immer wieder zu etablieren sucht. Schon kleinste Störungen im Mikro-Kosmos können beitragen, den Makro-Kosmos wieder im Urchaos versinken zu lassen, so z.B. das Vernachlässigen bzw. Ausbleiben der Riten oder das Erkranken eines Individuums (Schneider 2000: 48). Diese Rückführung in die geordnete Welt der Maat erfolgt mittels „Heka““ (hkk3) bzw. Analogie. Diese kann zum einen über die zu applizierenden Heilmittel und zum anderen über die Historiola geschehen. ${ }^{31}$ So wird mittels eines mythischen Präzedenzfalles auf das aktuelle Geschehen Bezug genommen, um einen identischen Ausgang der Situation (z. B. eine Heilung) zu schaffen. ${ }^{32}$ Die Historiola wirkt über die Sprache im Ritual. Demnach äußern die Worte des rezitierten Spruches ihre innewohnende Kraft in die Welt gegen eine Bedrohung. Dabei wird das götterweltliche Geschehen aktualisiert und in die menschliche Ebene transformiert. Dies wird durch die Gleichsetzung des Heilers und/oder des Patienten mit einer Gottheit erreicht bzw. durch die Gleichstellung des Vorfalls mit dem mythischen Vorbild.

\section{Coping-Strategie II}

Eine Wirkweise der Texte sieht Stegbauer (2015: 5-92) in einer möglichen Hypnose. So soll die Hypnoseindukti-

che (2015: 75). Die Vorstellung, dass die Mythen ad hoc ausgedacht wurden, war u. a. die Vorstellung von Altenmüller 1980, Sp. 1155; Eschweiler 1994: 240 und wurde u. a. von Quack 1996: 333 widerlegt. Zusammenfassend bei Stadler 2009.

${ }^{31}$ Leitz 2005: 41-62 beschäftigt sich mit der Wirkweise der zu verwendenden Drogen der Rezepte (pḥr.t). Diese können zum einen dem Prinzip contraria contrariis und similia similibus folgen.

32 Schneider 2000: 60-75 unterscheidet dabei: a) Analogie des Zielobjektes, b) Analogie der Handlung, c) Analogie des Aktanten, d) Analogie des Sprechaktes, e) Analogie der Bezeichnung, f) Analogie zwischen Hilfsmitteln. 
on durch das Einbinden des Patienten mittels Historiola geschehen. Die Aufmerksamkeit des Patienten würde durch den Ritualablauf gefesselt und er würde auf eine Art Phantasiereise geschickt, die die Selbstheilungskräfte aktivieren soll. So werden Bilder z. B. des Löschens von Feuer (eines brennenden Schmerzes) beschrieben, um eine Schmerzlinderung zu erreichen. Dass dieser Vergleich der modernen Hypnosetherapie mit der Wirkung der altägyptischen Heilungssprüche auf »tönernen Füßen« steht, ist Stegbauer (2015: 90) dabei klar und sie zeigt die Unterschiede hinsichtlich der Aktivität des Patienten auf, die in den altägyptischen Heilungssprüchen nicht zu finden ist (Stegbauer 2015: 91). ${ }^{33}$

Die Historiolae und Ritualhandlungen können mit dem so genannten Placebo-Effekt und dem Coping-Prozess ${ }^{34}$ in Verbindung gebracht werden. Nunn (vgl. Nunn 2002: 97; auch Quack 1999: 456) erkennt in der Verwendung von magischen Sprüchen einen Heileffekt, der auf Suggestion und Erwartung beruht. Diesem schließe ich mich an, ist doch die Religion und Götterwelt allgegenwärtig in der Lebenswelt der Ägypter. Deshalb ist es nicht verwunderlich anzunehmen, dass ein Patient sich in der Hand eines Heilers und Priesters, der das benötigte Wissen aufbieten kann, um in direkten Kontakt mit der Götterwelt zu treten, wohl und geborgen fühlt, in der Erwartung geheilt zu werden. Die Götter werden angerufen, um dem Patienten zu helfen und dieser wird kraft Analogie der Historiola dem Schutz und der Hilfe der Götter überantwortet. Für einen Kranken ist es wichtig, Vertrauen zu dem Heiler oder Arzt aufzubauen. Denn nur so kann ein Wohl- oder Sicherheitsempfinden entstehen, das dem Heilungsprozess und den Selbstheilungskräften förderlich ist. Dabei ist das so genannte Coping von Bedeutung. Denn Heilerfolge sind ebenso in der heutigen Medizin von Emotionen, Einstellungen und Erwartungen, Hoffnungen und Ängsten eines Patienten abhängig und die Fähigkeit zur Mobilisierung heilsamer Emotionen spielt eine wichtige Rolle (vgl. Hoheisel 1995: 169; 183). Dabei liegt die Heilkraft in »der Geistverfassung des Patienten, nicht in der Richtigkeit oder Natur des Geglaubten.« (ebd. 1995: 182)

Als Coping kann das »Bemühen definiert werden, bereits bestehende oder erwartete Belastungen durch die Krankheit innerpsychisch (emotional/kognitiv) oder durch zielgerichtetes Handeln vorbeugend auszugleichen oder ihre Manifestation zu verarbeiten und zu meistern.« (Buddeberg \& Willi 1992: 486-487). Dies zeigt die Anwendbarkeit auf sowohl reaktive als auch präventive Sprüche.

Zum anderen wird sich der Hilfe göttlicher Mächte durch die Gleichsetzung des Patienten oder Heilers mit den göttlichen Äquivalenten versichert, wodurch eine

\footnotetext{
${ }^{33}$ Hierunter ist die Aufforderung: »Du atmest tief ein und aus« $\mathrm{zu}$ verstehen.

${ }^{34}$ Zur Forschung von religiösen Verarbeitungsstilen außerhalb der Ägyptologie siehe u. a. Murken/Müller 2007; Murken 1998; Murken 1997; Pargament 1988; Pargament 1997; Pargament 1992. Trotz der zeitlichen und gesellschaftlichen Unterschiede der modernen Coping-Forschung zum Alten Ägypten sind doch einige der hier herausgegriffenen Aspekte übertragbar.
}

Abgabe von Kontrolle erfolgt. Dies ist eine Möglichkeit der Krankheitsbewältigung, die als passives coping (vgl. Pargament et al. 1988) beschrieben wird. Sich dem Heiler und/oder Gott anzuvertrauen, lindert die Angst und unterstützt das Sicherheitsgefühl und positive Emotionen. Dabei ist neben den persönlichen Eigenschaften eines Heilers (freundliches, vertrauenerweckendes Auftreten, Status der Ausbildung etc.) ebenfalls dessen kulturell determinierte Rolle von Bedeutung, um bestimmte Erwartungen des Patienten hervorzurufen.

In der religionspsychologischen Coping-Forschung wird die verstärkte Hinwendung zur Religiosität in Krisensituationen, wie z. B. während schwerer Krankheiten, untersucht. Dabei wurde festgestellt, dass Religiosität Trost und Hoffnung spenden kann, damit problematische Lebenssituationen besser überstanden werden können (Murken/Müller 2007: 117-118:). Neben der altägyptischen Zuordnung der Ursachen einer Krankheit als durch einen Dämon oder Wiedergänger oder als Strafe eines Gottes initiiert, wird ebenso die Heilung durch göttliche Hilfe erwartet. Aktuelle Studien zeigen, dass positive religiöse Verarbeitungsmuster mit einer vertrauensvollen Gottesbeziehung einhergehen (Murken/Müller 2007: 118-119; Murken 1998).

Somit liegt die Wirkung der altägyptischen Heilungssprüche m. E. in der Beruhigung des Patienten und Verarbeitungshilfe der Stressoren. Nicht unmittelbar durch Hypnose, sondern durch Relativieren (vgl. Buddeberg \& Willi 1992: 48) kann eine schmerz- und angstlindernde Wirkung eintreten - z. B. im Sinne folgender Gedanken: »Wenn Horus dies überlebt hat, überlebe ich das ebenfalls und die Analogie/Gottheit/Heiler wird mir dabei helfen.« o. ä. Dies ist der Versuch, sich mit dem eigenen Schicksalsschlag abzufinden, indem sich der Patient mit dem Schicksal und der positiven Schicksalsbewältigung anderer, hier im Falle mythologischer Patienten, vergleicht. Dabei spielt die direkte Gleichsetzung des Unheils des Patienten mit dem der mythischen Situation und des betroffenen Gottes und die Umsetzung im Ritual eine wichtige Rolle, auch die Anrufung der Götter zur Hilfeleistung durch Bitten oder Drohen. Hier tritt die von Nunn (2002: 97) angenommene Erwartung und Suggestion als Wirkung der Heilungstexte als positive Komponenten einer Bewältigungsstrategie, also Coping, hervor, die den altägyptischen Patienten beruhigen.

\section{Literatur}

Altenmüller, H. (1977) Gliedervergottung. In: Lexikon der Ägyptologie, in 7 Bänden 1975-1992, hrsg. von Wolfgang Helck. Bd. II. Wiesbaden: Harrassowitz, Sp. 624-627.

Altenmüller, H. (1980) Magische Literatur. In: Lexikon der Ägyptologie, in 7 Bänden 1975-1992, hrsg. von Wolfgang Helck. Bd. III. Wiesbaden: Harrassowitz, Sp. 1151-1162.

Assmann, J. (1977) Die Verborgenheit des Mythos im Alten Ägypten. In: Göttinger Miszellen 25, S. 7-43.

Assmann, J. (1991/1984) Theologie und Frömmigkeit einer frühen Hochkultur. Stuttgart u. a.: Kohlhammer. 
Assmann, J. (1997) Magic and Theology in Ancient Egypt. In: Peter Schäfer/Hans G. Kippenberg (Hrsg.), Envisioning Magic. A Princeton Seminar and Symposium, Studies in the History of Religions LXXV. Leiden u. a.: Brill, S. 1-18.

Assmann, J. (1998) Mythos. In: Handbuch religionswissenschaftlicher Grundbegriffe, in 5 Bänden 1988-2001, hrsg. von Hubert Cancik et al. Bd. IV, Stuttgart u.a.: Kohlhammer, S. 179-200.

Assmann, J. (2002) Altägyptische Totenliturgien. Band 1 Totenliturgien in den Sargtexten des Mittleren Reiches. Supplemente zu den Schriften der Heidelberger Akademie der Wissenschaften, Philosophisch-Historische Klasse 14. Heidelberg: Universitätsverlag C. Winter.

Assmann, J. (2003/2001) Tod und Jenseits im Alten Ägypten, Sonderausgabe 2003. München: C. H. Beck.

Berger, P. L. (1973) Zur Dialektik von Religion und Gesellschaft. Frankfurt am Main: Fischer.

Borghouts, J. F. (1980) Magie. In: Lexikon der Ägyptologie, in 7 Bänden 1975-1992, hrsg. von Wolfgang Helck. Bd. III. Wiesbaden: Harrassowitz, Sp. 1137-1151.

Brawanski, A. (2001) Die Fälle 1-8 des Papyrus Edwin Smith (»Schädelhirntraumafälle«). In: Studien zur Altägyptischen Kultur 29, S. 7-39.

Buddeberg, C. \& Willi, J. (1998) Psychosoziale Medizin. 2. vollst. Überarbeitete Auflage. Berlin/Heidelberg: Springer.

Burkard, G. (2006) Drei Amulette für Neugeborene aus Elephantine. In: Gerald Moers u. a. (Hrsg.), jn.t dr.w: Festschrift für Friedrich Junge 1, Göttingen: Lingua Aegyptia, Seminar für Ägyptologie und Koptologie. S. 109-124.

Edwards, I. E. S. (1960) Hieratic Papyri in the British Museum. Fourth Series. Oracular Amuletic Decrees of the Late New Kingdom Edited, together with Supplementary Texts in other collections, 2. volumes, London, Published by Trustees of the British Museum.

Erman, A. (1934) Die Religion der Ägypter: ihr Werden und Vergehen in vier Jahrtausenden. Berlin, De Gruyter.

Eschweiler, P. (1994) Bildzauber im alten Ägypten: die Verwendung von Bildern und Gegenständen in magischen Handlungen nach den Texten des Mittleren und Neuen Reiches. Orbis Biblicus et Orientalis 137. Freiburg u. a.: Universitätsverlag; Vandenhoeck \& Ruprecht.

Fischer-Elfert, H.-W. (2005a) Pap. Ebers Nr. 1-3: Reflexion eines altägyptischen Heilers über seine Initiation? In: Hans-W. Fischer-Elfert (Hrsg.), Papyrus Ebers und die antike Heilkunde: Akten der Tagung vom 15.16.3.2002 in der Albertina/UB der Universität Leipzig. Wiesbaden: Harrassowitz, S. 133-147.

Fischer-Elfert, H.-W. (2005b) Altägyptische Zaubersprüche. Reclams Universal-Bibliothek 18375. Stuttgart: Reclam.

Fischer-Elfert, H.-W. (2005c) Abseits von Ma'at: Fallstudien zu Außenseitern im alten Ägypten. Wahrnehmungen und Spuren Altägyptens 1. Würzburg: Ergon.

Fischer-Elfert, H.-W. (2007) Heilkunde im alten Ägypten. In: Harald Froschauer/Cornelia Römer (Hrsg.), Zwi- schen Magie und Wissenschaft: Ärzte und Heilkunst in den Papyri aus Ägypten. Wien: Phoibos, S. 43-54.

Fischer-Elfert, H.-W. (2015) Magika hieratika in Berlin, Hannover, Heidelberg und München. Ägyptische und Orientalische Papyri und Handschriften des Ägyptischen Museums und Papyrussammlung Berlin 2. Berlin, De Gruyter.

Fitzenreiter, M. (1994) Zum Ahnenkult in Ägypten. In: Göttinger Miszellen 143, S. 51-72.

Frankfurter, D. (1995) Narrating Power: the theory and practice of the magical historiola in ritual spells. In: Marvin Meyer/Paul Mirecki (Hrsg.) Ancient magic and ritual power. Religions in the Graeco-Roman world 129. Leiden u. a.: Brill, S. 457-476.

Ghalioungui, P. (1983) The Physicians of Pharaonic Egypt. Deutsches Archäologisches Institut Kairo, Sonderheft 10. Cairo: Al-Ahram Center for Scientific Translations/Mainz: von Zabern.

Girard, R. (1998) Der Sündenbock. Zürich: Benziger.

Gutekunst, W. (1987) Wie »magisch« ist die »Magie« im alten Ägypten? Einige theoretische Bemerkungen zur Magie-Problematik. In: Alessandro Roccati \& Alberto Siliotti (Hrsg.), La magia in Egitto ai tempi dei faraoni: atti, convegno internazionale di studi, Milano, 29-31 ottobre. Verona: Rassegna internazionale di cinematografia archeologica arte e natura libri. S. 77-98.

Gutekunst, W. (1995) Textgeschichtliche Studien zum Verjüngungsspruch (Text B) auf Horusstelen und Heilstatuen, Trier: Selbstverlag.

Haas, V. (2002) Hethitische Heilverfahren. In: Axel Karenberg/Christian Leitz (Hrsg.) 2002. Heilkunde und Hochkultur II: »Magie und Medizin« und »Der alte Mensch « in den antiken Zivilisationen des Mittelmeerraumes. Naturwissenschaft, Philosophie, Geschichte 16. Münster u. a.: LIT-Verlag. S. 21-48.

Halioua, B. \& Ziskind, B. (2005) Medicine in the days of the pharaohs. Cambridge u. a.: Belknap.

Hoheisel, K. (1995) Religiöse und profane Formen nichtmedizinischen Heilens. In: Karl Hoheisel/Hans-Joachim Klimkeit (Hrsg.), Heil und Heilung in den Religionen. Wiesbaden: Harrassowitz, S. 167-184.

Harrison, M. O. et al. (2001) The epidemiology of religious coping: a review of recent literature. In: International Review of Psychiatry 13, S. 86-93.

Kessler, D. (2005) Zur Funktion der Horusstelen und Heilstatuen vor den Sanktuaren und Kapellen. In: Khaled Daoud, u. a. (Hrsg.), Studies in Honor of Ali Radwan 2, Le Caire: Conseil Suprême des Antiquités, S. 81-94.

Kolta, K. S. \& Schwarzmann-Schafhauser, D. (2000) Die Heilkunde im alten Ägypten: Magie und Ratio in der Krankheitsvorstellung und therapeutischen Praxis. Sudhoffs Archiv. Beihefte 42. Stuttgart: Franz Steiner.

Leitz, C. (2002a) Die medizinischen Texte aus dem Alten Ägypten. In: Axel Karenberg/Christian Leitz (Hrsg.), Heilkunde und Hochkultur I: Geburt, Seuche und Traumdeutung in den antiken Zivilisationen des Mittelmeerraumes, Münster u. a.: LIT-Verlag, S. 17-34.

Leitz, C. (2002b) Rabenblut und Schildkrötengalle: zum 
vermeintlichen Gegensatz zwischen magisch-religiöser und empirisch-rationaler Medizin. In: Axel Karenberg/ Christian Leitz (Hrsg.), Heilkunde und Hochkultur II: »Magie und Medizin« und »Der alte Mensch« in den antiken Zivilisationen des Mittelmeerraumes, Münster u. a.: LIT-Verlag, S. 49-73.

Leitz, C. (2005) Die Rolle von Religion und Naturbeobachtung bei der Auswahl der Drogen im Papyrus Ebers. In: Hans-W. Fischer-Elfert (Hrsg.), Papyrus Ebers und die antike Heilkunde: Akten der Tagung vom 15.16.3.2002 in der Albertina/UB der Universität Leipzig, Wiesbaden: Harrassowitz. S. 41-62.

Manisali, A. (2004) »Imitate but innovate« oder: Eine Götterbedrohung mit hymnischer Struktur im Papyrus Genf MAH 15274. In: Studien zur Altägyptischen Kultur 32, S. 301-309.

Morenz, L. (1997) (Magische) Sprache der »geheimen Kunst«. In: Studien zur Altägyptischen Kultur 24, S. 191201.

Murken, S. (1997) Ungesunde Religiosität - Entscheidungen der Psychologie? In: Gritt Maria Klinkhammer (Hrsg.) Kritik an Religionen: Religionswissenschaft und der kritische Umgang mit Religionen. Marburg: Diagonal-Verlag, S. 157-172.

Murken, S. (1998) Gottesbeziehung und psychische Gesundheit: Die Entwicklung eines Modells und seine empirische Überprüfung. Münster: Waxmann.

Murken, S. \& Müller, C. (2007): »Gott hat mich so ausgestattet, dass ich den Weg gehen kann.« Religiöse Verarbeitungsstile nach der Diagnose Brustkrebs. In: Lebendiges Zeugnis 62, S. 115-128.

Nunn, J. F. (2002/1996) Ancient Egyptian Medicine. London: British Museum Press.

Pargament, K. I. et al. (1988) Religion and the problemsolving process: Three styles of coping. In: Journal for the Scientific Study of Religion 27, S. 90-104.

Pargament, K. I. (1992) Religion and Prevention in Mental Health: Research, Vision, and Action. New York u. a.: Haworth.

Pargament, K. I. (1997) The Psychology of Religion and Coping: Theory, Research, Practice. New York u. a.: Guilford Press.

Pargament, K. I., et al. (2006): Spiritual Resources and Struggles in Coping with Medical Illness. In: Southern Medical Journal 99(10), S. 1161-1162.

Pommerening, T. (2005) Review: Kolta, Kamal Sabri and Doris Schwarzmann-Schafhauser 2000. Die Heilkunde im alten Ägypten: Magie und Ratio in der Krankheitsvorstellung und therapeutischen Praxis. Sudhoffs Archiv. Beihefte 42. Berichte zur Wissenschaftsgeschichte 28 (4), Stuttgart, S. 353-354.

Quack, J. F. (1992) Studien zur Lehre für Merikare. Göttinger Orientforschungen, 4. Reihe: Ägypten 23. Wiesbaden: Harrassowitz.

Quack, J. F. (1995) Dekane und Gliedervergottung: altägyptische Traditionen im Apokryphon Johannis. In: Jahrbuch für Antike und Christentum 38, S. 97-122.

Quack, J. F. (1996) Das Pavianshaar und die Taten des
Thot (pBrooklyn 47.218.48+85 3,1-6). In: Studien zur Altägyptischen Kultur 23, S. 305-333.

Quack, J. F. (1999) Westendorf, Wolfhart: Handbuch der altägyptischen Medizin. Handbuch der Orientalistik, Erste Abteilung: Der Nahe und Mittlere Osten. Sechsunddreißigster Band. Leiden/Boston/Köln: Brill 1999. - Besprochen von Joachim Friedrich Quack, Berlin. In: Orientalistische Literaturzeitung 64, Berlin, S. 455-462.

Quack, J. F. (2003) Methoden und Möglichkeiten der Erforschung der Medizin im Alten Ägypten. Medizinhistorisches Journal 38 (1), S. 3-15.

Quack, J. F. (2005) Tabuisierte und ausgegrenzte Kranke nach dem »Buch vom Tempel«. In: Hans-W. Fischer-Elfert, (Hrsg.), Papyrus Ebers und die antike Heilkunde: Akten der Tagung vom 15.-16.3.2002 in der Albertina/ UB der Universität Leipzig, Wiesbaden: Harrassowitz, S. 63-80.

Raffel, J. (2009) Die Rolle des Gottes Horus als Arzt und Patient in den sog. Magischen Texten, unpubl., Magisterarbeit Universität Leipzig.

Raffel, J. (2019) Egal was, ich war's nicht - Zur NichtIdentifikationsformel in Zusammenhang mit der Götterbedrohung. In: Marc Brose/Peter Dils/Franziska Naether/ Lutz Popko/Dietrich Raue (Hrsg.), En détail - Philologie und Archäologie im Diskurs. Festschrift für Hans-W. Fischer-Elfert II, ZÄS Beiheft 7, S. 947-964.

Ritner, R. K. (1993) The Mechanics of ancient Egyptian magical practice. Studies in Ancient Oriental Civilization 54. Chicago: The Oriental Institute of the University of Chicago.

Rost, A. (2008) Die Ermordung des Gottes Osiris aus der Perspektive der Sündenbocktheorie von René Girard, unpubl., Magisterarbeit Universität Leipzig.

Schneider, T. (2000) Die Waffe der Analogie: Altägyptische Magie als System. In: Karen Gloy/Manuel Bachmann (Hrsg.), Das Analogiedenken: Vorstöße in ein neues Gebiet der Rationalitätstheorie, Freiburg: Karl Alber. S. 37-85.

Stadler, M. A. (2009) Weiser und Wesir. Studien zum Vorkommen, Rolle und Wesen des Gottes Thot im ägyptischen Totenbuch, Orientalische Religionen in der Antike 1, Tübingen: Mohr Siebeck.

Stegbauer, K. (2012) Dialog und Monolog als Ausdrucksmittel magischer Texte. In: Amr El Hawary (Hrsg.), Wenn Götter und Propheten reden - Erzählen für die Ewigkeit. Studien des Bonner Zentrums für Transkulturelle Narratologie. Narratio Aliena? 3, Berlin: EB-Verlag, S. 172-184.

Stegbauer, K. (2015) Magie als Waffe gegen Schlangen in der ägyptischen Bronzezeit. Borsdorf: Ed. Winterwork.

Sternberg-El Hotabi, H. (1999) Untersuchungen zur Überlieferungsgeschichte der Horusstelen: ein Beitrag zur Religionsgeschichte Ägyptens im 1. Jahrtausend v. Chr.; In: Ägyptologische Abhandlungen 62. Wiesbaden: Harrassowitz.

Weber, S. R. \& Pargament, K. I. (2014) The role of religion and spirituality in mental health. In: Current Opinion in Psychiatry 27(5), S. 358-363 
Wente, E. F. (1967): Late Ramesside Letters, Chicago/ Illinois: The University of Chicago Press $=$ The Oriental Institute of Chicago. In: Studies in Ancient Oriental Civilization 33.

Westendorf, W. (1992) Erwachen der Heilkunst. Die Medizin im Alten Ägypten. Zürich/München: Artemis \& Winkler.

Westendorf, W. (1999) Handbuch der Altägyptischen Medizin, 1.-2. Band, Leiden u. a.: Brill, $1999=$ Handbuch der Orientalistik/ Handbook of Oriental Studies. Erste Abteilung: Der Nahe und Mittlere Osten/ The Near and Middle East, 36/1.
Westendorf, W. (2010) Texte aus Ägypten: 1. Die altägyptische Medizin. In: Bernd Janowski/Daniel Schwemer (Hrsg.), Texte zur Heilkunde, Gütersloh: Gütersloher Verlagshaus Mohn, S. 195-216.

\section{Internetquellen}

Fischer-Elfert, Hans-W. (2006): Magie in Ägypten. In: Das wissenschaftliche Bibellexikon im Internet, www.wibilex.de, letzter Zugriff: 30.04.2009.

WHO: http://www.euro.who.int/de/about-us/organization/who-worldwide, letzter Zugriff: 05.03.2015. 